INPLASY

PROTOCOL

To cite: $X v$ et al. Do strength trainings influence ankle functions in patients with chronic ankle instability ? A systematic review with metaanalyses. Inplasy protocol

202110032. doi:

10.37766/inplasy2021.1.0032

Received: 11 January 2021

Published: 12 January 2021

Corresponding author:

Xiaoyun Xv

nmuxvxiaoyun@163.com

Author Affiliation:

Shanghai University of Sport

Support: NNS Found of China.

Review Stage at time of this submission: The review has not yet started.

Conflicts of interest: None.

\section{Do strength trainings influence ankle functions in patients with chronic ankle instability ? A systematic review with meta-analyses}

Xv, X1; Xue, X2; Hua, Y3.

Review question / Objective: Do strength trainings influence ankle functions(such as ankle function tests or questionaires) in patients with chronic ankle instability?

Condition being studied: Lateral Ankle sprain (LAS) is one of the most common sport-related lower extremity musculoskeletal injury. Over 2 million ankle sprains are treated in emergency departments in the US and UK each year, resulting in about $\$ 2$ billion of healthcare costs. In longterm prognosis, more than $30 \%$ of patients reporting repetitive bouts of ankle giving way and recurring sprains, termed as chronic ankle instability (CAI). For the patients suffering from persistent symptoms, surgery is usually suggested and most of them can obtain good outcomes, while some still end up poorly and fail to return to sport. One possible can be the sensorimotor deficits, which might be enhanced by functional trainings.

INPLASY registration number: This protocol was registered with the International Platform of Registered Systematic Review and Meta-Analysis Protocols (INPLASY) on 12 January 2021 and was last updated on 12 January 2021 (registration number INPLASY202110032).

\section{INTRODUCTION}

Review question / Objective: Do strength trainings influence ankle functions(such as ankle function tests or questionaires) in patients with chronic ankle instability?
Rationale: The results of studies on the effects of strength training on ankle functions in CAl patients were in conflicts.

Condition being studied: Lateral Ankle sprain (LAS) is one of the most common sport-related lower extremity 
musculoskeletal injury. Over 2 million ankle sprains are treated in emergency departments in the US and UK each year, resulting in about $\$ 2$ billion of healthcare costs. In longterm prognosis, more than $\mathbf{3 0}$ $\%$ of patients reporting repetitive bouts of ankle giving way and recurring sprains, termed as chronic ankle instability (CAI). For the patients suffering from persistent symptoms, surgery is usually suggested and most of them can obtain good outcomes, while some still end up poorly and fail to return to sport. One possible can be the sensorimotor deficits, which might be enhanced by functional trainings.

\section{METHODS}

Search strategy: Search strategies for PubMed in title or abstract :(a):"ankle injuries" [MESH] OR unstable OR instabilit* OR strain* OR sprain* OR rupture* OR tear* OR chronic ankle instability OR ankle

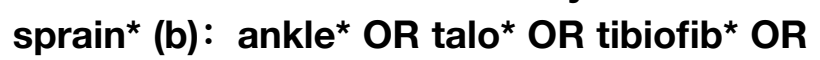
tibio-fib* OR "lateral ligament" OR "lateral ligaments" OR ankle joint* OR talocrural OR talo-crural OR talo-calcaneal OR "Lateral Ligament, Ankle"[MESH] (c) : Strength Training OR strengthening exercise OR Muscle strengthening OR LP BAND OR "Resistance Training/therapeutic us e "[ M e sh] OR "Endurance Training"[Mesh]. Formal search strategy is "(a) AND (b) AND (c)".

Participant or population: Individuals with chronic ankle instability.

Intervention: Ankle strength training.

Comparator: Ankles without strength training.

Study designs to be included: Crossover or cohort or RCT.

Eligibility criteria: Peer-reviewed human studies in English that investigated the effect of strength trainings on ankle functions in the injured ankle of individuals with a history of ankle sprain.
Information sources: Five electronic database, including Embase, Web of Science, SPORTDiscus, PubMed, and Cochrane Library. The reference lists of each included paper were also checked manually.

Main outcome(s): Ankle functional tests or questionnaires of the injured ankles.

Additional outcome(s): None.

Data management: Studies were reviewed independently by two authors (XXY, XXA). If disagreements couldn't be resolved through discussion, the third reviewer (HYH) was consulted. Then, the same procedure was applied to extract following information: demographic data, sample size, description of trainings, details of test methodology (e.g. movement direction, target angle, angular velocity), joint proprioception results (means and standard deviation), and summarized results. The authors were contacted if numerical data were confusing or not reported.

Quality assessment / Risk of bias analysis: All the authors discussed the standard of each item before formal rating, and two authors (XXY, XXA) rated the included studies independently. The inter-rater agreements of the initial ratings were calculated, and the third reviewer (HYH) be consulted for disagreements. To assess the quality of studies, the Methodological index for non-randomized studies (MINORS) was applied. To assess the variability of CAI patients, the recommendation of the International Ankle Consortium was applied. The standard CAl criteria included: (1) At least 1 significant ankle sprain that occurred at least 12 months ago and resulted in pain, swelling and at least 1 interrupted day of desired physical activity; (2) Do not have any ankle injury in the past 3 months; (3) Have at least 1 of the classical symptoms.

Strategy of data synthesis: A pair-wised or network meta-analysis of the randomeffects model would be performed for the studies similar in strength training type and 
movement direction. Standardized mean difference (SMD) was calculated for all data between CAl patients and controls with 95\% confidence intervals (Cl). Higher SMD represented larger ankle function improvement in CAl, with $0.2 \sim 0.5$ as weak, $0.5 \sim 0.8$ as moderate, $>0.8$ as large-sized effect. To evaluate heterogeneity, $Q$ and 12 statistics were calculated, with $\mathrm{p}<0.05$ as statistically significant and $I^{\wedge} 2$ values $\geq 75 \%$ as high heterogeneity.

Subgroup analysis: None.

Sensibility analysis: Sensitivity analysis would be conducted through removing single study at a time and then evaluating the pooled results again.

Language: English.

Country(ies) involved: China.

Other relevant information: None.

Keywords: strength training, chronic ankle instability, meta-analysis.

Dissemination plans: Published in SCI journals.

Contributions of each author:

Author 1 - Xiaoyun Xv - Study design; literature search and selection; data collection; quality rating; statistical analysis; writing of the manuscript.

Email: 16301050010@fudan.edu.cn

Author 2 - Xiao'ao Xue - Study design; literature search and selection; data collection; quality rating; statistical analysis; reviewing of the manuscript.

Email: hua_cosm@aliyun.com

Author 3 - Yinghui Hua - Supervision of literature search, data collection and quality rating; reviewing the manuscript. 